\title{
Effect of implant temperature on secondary defects created by MeV Sn implantation in silicon
}

\author{
J. Wong-Leung, a) C. Jagadish, and M. J. Conway \\ Department of Electronic Materials Engineering, Research School of Physical Sciences and Engineering, \\ The Australian National University, Canberra, ACT 0200, Australia \\ J. D. Fitz Gerald \\ Petro Physics Group, Research School of Earth Sciences, The Australian National University, \\ Canberra, ACT 0200, Australia
}

(Received 18 September 2000; accepted for publication 15 November 2000)

\begin{abstract}
Secondary defects induced by ion implantation in silicon after annealing have been previously shown to vary with the implantation and annealing conditions. However, in the low dose implants, well below the amorphization dose, the defects have been predominantly characterized to be interstitial in nature. In this article, we study the effect of implant temperature on secondary defects created by $1 \mathrm{MeV}$ Sn implantation to a dose of $3 \times 10^{13} \mathrm{~cm}^{-2}$ after subsequent annealing. We report a variation in the defect microstructure with implant temperature showing preferential formation of small interstitial loops for $-191{ }^{\circ} \mathrm{C}$ and only rod-like defects for similar implants carried out at $300{ }^{\circ} \mathrm{C}$. We conclude that these microstructures are a result of the dense cascades created by heavy Sn ions, creating local amorphous pockets in the implant damage region at the lowest implant temperatures. The variation of the microstructure with implant temperature is interpreted in terms of the effect of dynamic annealing over the defects formed in silicon. (c) 2001 American Institute of Physics. [DOI: 10.1063/1.1340602]
\end{abstract}

\section{INTRODUCTION}

Ion implantation constitutes an important part of semiconductor processing leading to the fabrication of integrated circuits. The reduction in device size is creating a need for fundamental research with an aim to create appropriate computer simulations, capable of predicting ion implantation induced defects and their role in dopant diffusion. The various implantation and annealing parameters influencing defect formation still need to be studied and their effect understood. The damage created by ion implantation into silicon generally anneal out but above a certain dose known as the critical dose, dependent on the annealing temperature, extended defects are usually formed. Various articles have studied this critical dose using transmission electron microscopy (TEM) ${ }^{1}$ deep level transient spectroscopy, ${ }^{2}$ or both techniques ${ }^{3,4}$ and photoluminescence., ${ }^{5,6}$ A range of extended defects (for a review see Ref. 7) induced by ion implantation have been reported in Si, namely $\{113\}$ rod-like defects, ${ }^{8-10}$ faulted Frank loops, perfect loops, $\{111\}$ rod-like defects, ${ }^{11}$ and voids. The $\{113\}$ defects have the highest energy configuration and have been shown to unfault into Frank $a / 3\langle 111\rangle$ loops. ${ }^{12}$ The latter have been well known to unfault into perfect loops of $a / 2\langle 110\rangle$ which have the lowest energy configuration. The defects observed in Si have been primarily extrinsic defects, i.e., interstitial in nature except for voids and some intrinsic loops observed in $\mathrm{Si}$ substrate in $\mathrm{GeSi} / \mathrm{Si}$ layers. ${ }^{13}$

The Frenkel pairs generated by the collision cascades during implantation are believed to anneal out, leaving one extra ion injected from implantation, described as the +1

${ }^{a)}$ Electronic mail: jw1109@rsphysse.anu.edu.au model. Several studies ${ }^{1,2,14}$ have revealed some discrepancies in the +1 model and Schreutelkamp et al. ${ }^{1}$ have proposed that the criterion for secondary defect formation is the total number of displaced silicon exceeding a critical value. This can explain the general trend observed for the difference observed in the critical dose with the mass of the implanted ion. ${ }^{2-4}$ Furthermore, transient enhanced diffusion (TED) was observed to be different for different implanted species making the $+n$ model a more universal formulation for simulating TED of B due to implantation damage. ${ }^{15,16}$

\section{EXPERIMENT}

Czochralski $p$-type Si wafers of (100) orientation were implanted with $1 \mathrm{MeV}$ Sn to a dose of $3 \times 10^{13} \mathrm{~cm}^{-2}$ at various implant temperatures ranging from -191 to $300{ }^{\circ} \mathrm{C}$. The range of $1 \mathrm{MeV}$ Sn implant is around $4000 \AA$ and a dose of $3 \times 10^{13} \mathrm{Sn} \mathrm{cm}^{-2}$ is well below the amorphization threshold for Sn implants in Si. The samples were then annealed at $800{ }^{\circ} \mathrm{C}$ for $15 \mathrm{~min}$. Cross-sectional samples with foil normal close to the $\langle 011\rangle$ direction were prepared by mechanical polishing and ion beam thinning using a Gatan duo mill. The latter was operated at $4 \mathrm{kV}$ using room temperature and liquid nitrogen stages for annealed and as-implanted samples, respectively. Plan view samples were prepared by conventional chemical etching. Both cross-sectional and plan view samples were examined by TEM using a Philips EM430 instrument and a Philips CM300 operated at 300 and $200 \mathrm{keV}$, respectively. The convention used for the coordinate system is defined with the implant surface normal as [100] and the cross-section sample foil normal as [011]. Trace analysis was used to determine the habit plane of loops. Defects were 


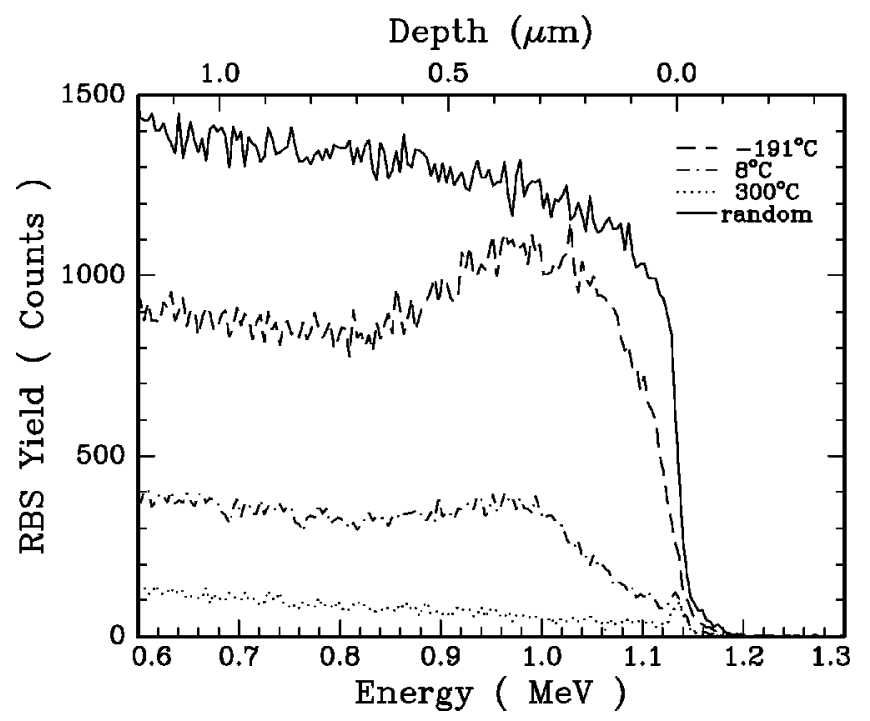

FIG. 1. RBSC spectra of Si wafers as-implanted with $1 \mathrm{MeV}$ Sn to a dose of $3 \times 10^{13} \mathrm{~cm}^{-2}$ with implant temperatures with the random level indicated in solid line.

imaged using various $g$ 's and the Burgers vector of the defects determined with the invisibility criteria satisfied for at least 2 reflections wherever possible. Rutherford backscattering and channeling (RBSC) was carried out on both asimplanted and annealed samples to assess any differences in the damage or stress around the implant region.

\section{RESULTS AND DISCUSSION}

Figure 1 shows the channeling RBS spectra of the asimplanted samples implanted with $1 \mathrm{MeV}$ Sn ions to a dose of $3 \times 10^{13} \mathrm{~cm}^{-2}$ at different implant temperatures. The sample implanted with $\mathrm{Sn}$ at $-191{ }^{\circ} \mathrm{C}$ shows a high dechanneling level at the implant depth which is close to the random height. With increasing implant temperature, the dechanneling occurring at the implant depth decreases indicating that the amount of damage created by implantation was significantly lower for the samples implanted at higher temperatures. The samples implanted at an elevated temperature of $300{ }^{\circ} \mathrm{C}$ had a channeling level close to the virgin silicon. The sample implanted at $-191{ }^{\circ} \mathrm{C}$ was also examined by cross-sectional transmission electron microscopy (XTEM) to ascertain the extent of amorphization in the asimplanted sample. We observe a speckled band at a depth of $\approx 1750-3500 \AA$ presumably due to lattice strains around many tiny amorphous regions created by the Sn implant. Furthermore, thickness fringes were visible near the surface of the sample confirming that the implanted layer did not become completely amorphous.

Dislocation loops were present in some of the annealed samples [see Figs. 2(a), 2(b), 3(a), and 3(b)]. Two types of loops were observed, both with a $\{111\}$ habit plane: (a) perfect loops with Burgers vector of type $a / 2\langle 011\rangle$ oriented at an angle of $54.8^{\circ}$ to the loop plane (examples are marked with a P prefix) (b) faulted Frank loops with Burgers vector of type $a / 3\langle 111\rangle$ perpendicular to the loop plane (examples are marked with an F prefix). Diffraction contrast experiments were required to differentiate between perfect and

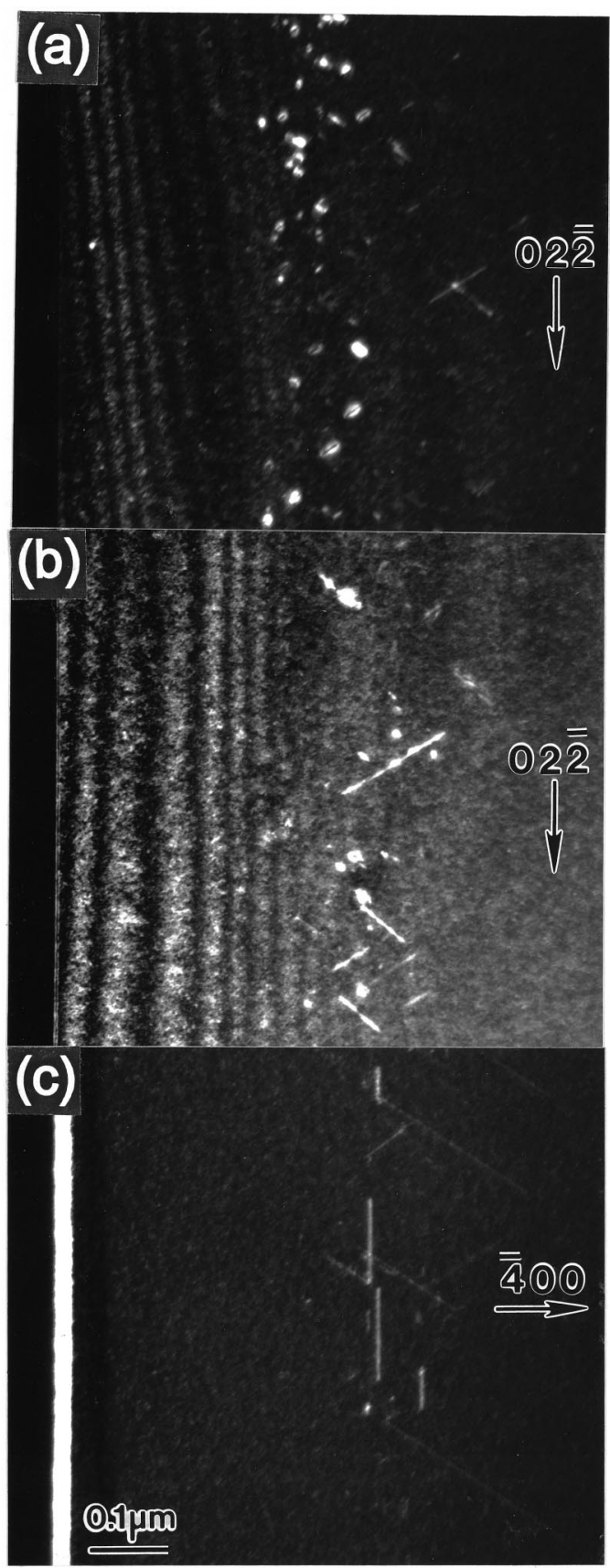

FIG. 2. XTEM micrographs of Si wafers implanted with $1 \mathrm{MeV} \mathrm{Sn}$ to a dose of $3 \times 10^{13} \mathrm{~cm}^{-2}$ after annealing at $800^{\circ} \mathrm{C}$ for $15 \mathrm{~min}$ with different implant temperatures (a) $-191{ }^{\circ} \mathrm{C}$, (b) $8{ }^{\circ} \mathrm{C}$, and (c) $300{ }^{\circ} \mathrm{C}$.

faulted loops and used two distinct image characteristics: (1) Perfect loops showed no internal contrast since they contain no internal stacking fault. However, faulted loops can have very weak stacking fault contrast under certain imaging conditions. ${ }^{17}$ As a result, confusion was avoided by recording images using both forward and reverse $\mathbf{g}$ with several different $\mathbf{g} .{ }^{18}$ (2) The perfect loops always showed stronger diffraction contrast than faulted loops because perfect loops generally had a higher $\mathbf{g} \cdot \mathbf{b}$ value due to their Burgers vectors being longer.

We could not apply the criterion used in other studies ${ }^{19}$ to separate perfect loops from faulted loops on the basis of shape since none of our loops appear faceted. We established 


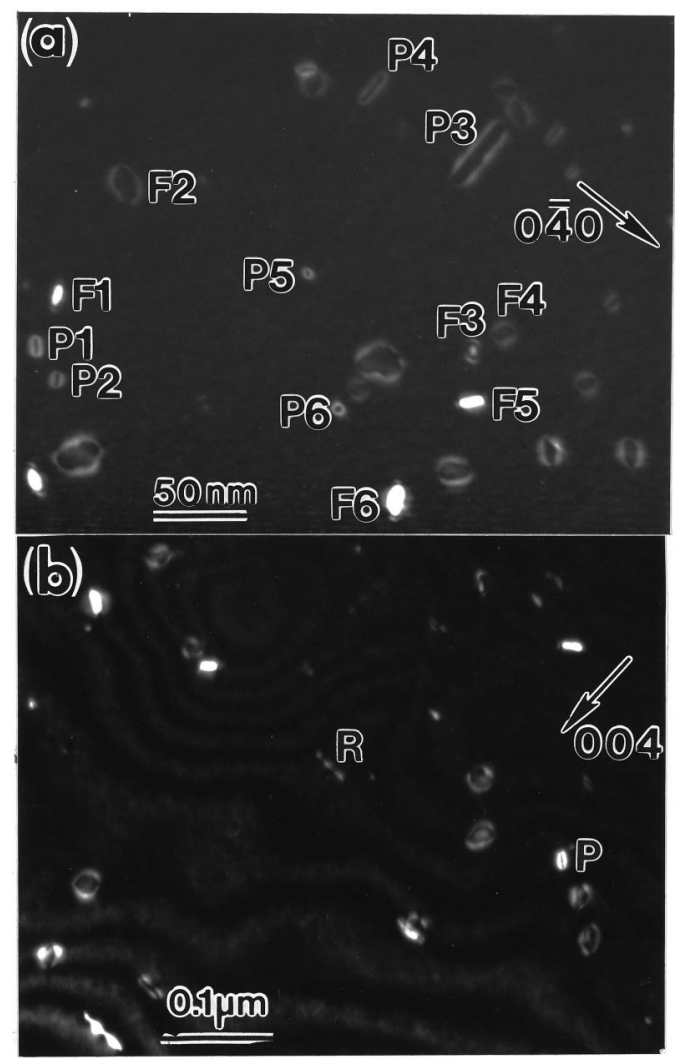

FIG. 3. PTEM micrograph of Si wafers implanted with $1 \mathrm{MeV}$ Sn to a dose of $3 \times 10^{13} \mathrm{~cm}^{-2}$ after annealing at $800^{\circ} \mathrm{C}$ for $15 \mathrm{~min}$ with implant temperature (a) $-191{ }^{\circ} \mathrm{C}$ and (b) $8{ }^{\circ} \mathrm{C}$.

that the small perfect loops in our samples were rounded and not of the hexagonal shapes reported elsewhere. ${ }^{19,20}$ Indeed, Jenkins et al. ${ }^{17}$ observed faceting of loop edges parallel to $\langle 110\rangle$ directions for loop sizes of $100 \AA$, but not for loop sizes $<50 \AA$. The lack of faceting for smaller loops can be explained by an Ostwald ripening process similar to the one observed for precipitates. ${ }^{21,22} \mathrm{We}$ also point out that our $g / 3 g$ weak-beam images using 200 or $300 \mathrm{keV}$ electrons with inherently smaller values of deviation parameter would be a little less sensitive to the presence of very small facets in comparison to the study of Jenkins et al. ${ }^{17}$ where $100 \mathrm{keV}$ electrons were used. For faulted loops, we note that the absence of faceting or hexagonal shapes is likewise in contrast to faulted loops observed in our own earlier studies. ${ }^{4}$

The annealed sample implanted at $-191{ }^{\circ} \mathrm{C}$ analyzed by RBS showed a channeling spectrum identical to the virgin silicon, clearly indicating that the damaged silicon was substantially recrystallized by annealing. This sample was also studied by both XTEM and plan view transmission electron microscopy (PTEM) in detail. Figure 3(a) shows a band of small loops at a depth of $3000 \AA$ and rod-like defects at a depth of $5000 \AA$ A. From the PTEM study of a large number of loops (see Fig. 3), their average diameter is $140 \AA$ and standard deviation $40 \AA$. The faulted loops are generally circular in shape and are unfaceted while the perfect loops showed preferential elongation along one $\langle 110\rangle$ direction. As an example, in Fig. 3(a), it is clear that P3 and P4 have an elongation much larger than that expected from the $\{111\}$ loops' inclination to the (100) foil. As observed in Ref. 20, such
TABLE I. Defect characteristics in Si samples implanted at various temperatures with $1 \mathrm{MeV}$ Sn ions.

\begin{tabular}{rccl}
\hline \hline $\begin{array}{c}\text { Implant } \\
\text { temperature } \\
\left({ }^{\circ} \mathrm{C}\right)\end{array}$ & Loop diameter $(\AA)$ & $\%$ of faulted loops & \multicolumn{1}{c}{ RLDs } \\
\hline-191 & $140 \pm 40$ & $50 \%$ & Some \\
8 & $220 \pm 50$ & $90 \%$ & Some \\
300 & No loops & $\cdots$ & Yes, RLDs only \\
\hline \hline
\end{tabular}

RLDs-rod-like defects

perfect loops have a Burgers vector along that $\langle 110\rangle$ perpendicular to the $\langle 110\rangle$ direction of elongation. This particular configuration of the loop made it harder to satisfy the true invisibility criterion, i.e., both $\mathbf{g} \cdot \mathbf{b}$ and $\mathbf{g} \cdot \mathbf{b} \times \mathbf{u}$ zero. For instance, loop P3 has a ( $\overline{1} 1 \overline{1})$ habit plane with a [101] elongation direction and a [101] Burgers vector. In Fig. 3(a), the imaging $\mathbf{g}$ is $0 \overline{4} 0$ and $\mathrm{P} 3$ is visible even though $\mathbf{g} \cdot \mathbf{b}=\mathbf{0}$ as $\mathbf{g} \cdot \mathbf{b} \times \mathbf{u} \neq \mathbf{0}$ especially for the long segments of the loop. True invisibility was only achieved for P3 when imaged with $\mathbf{g}=20 \overline{2}$ at the $[\overline{1} 1 \overline{1}]$ zone axis when both the $\mathbf{g} \cdot \mathbf{b}$ and $\mathbf{g} \cdot \mathbf{b} \times \mathbf{u}$ are zero. In this sample $\left(-191{ }^{\circ} \mathrm{C}\right)$, about $50 \%$ of the loops are faulted. All loops are interstitial as confirmed by conventional characterization procedures. ${ }^{20}$

XTEM and PTEM examination of the sample implanted at $8{ }^{\circ} \mathrm{C}$ after annealing also showed the formation of faulted loops, perfect loops, and rod-like defects. However, in this sample the loops are located further from the surface than those in the $-191{ }^{\circ} \mathrm{C}$ sample [see Fig. 2(b)]. Perfect loops were observed as deep as $6750 \AA$ which corresponds to the depth of the end of range of the implant. Figure $3(b)$ shows a typical image of the plan view sample implanted at $8{ }^{\circ} \mathrm{C}$. The circular loops are predominantly faulted Frank loops, while the $a / 2\langle 110\rangle$ elongated loops are perfect. In contrast to the sample implanted at $-191^{\circ} \mathrm{C}$, the loops have a larger average diameter of $220 \AA$ and standard deviation of $50 \AA$. About $90 \%$ of the loops are faulted [Figure 3(b)]. All loops are interstitial.

The sample implanted at an elevated temperature of $300{ }^{\circ} \mathrm{C}$ was also studied by TEM. Both cross-sectional [Fig. $2(\mathrm{c})]$ and plan view samples showed $\{311\}$ rod-like defects but no loops. The RBS spectrum of this annealed sample shows that the implanted layer has recrystallized very well and confirms the limitations of RBS to determine the type of defects created. ${ }^{7}$

The dramatic difference in microstructure (see Table I) resulting from the implant temperature difference needs explaining, particularly since such a large difference in microstructure has been produced entirely by change in implant temperature in the subamorphous dose regime. We did not observe such a difference in the microstructure with implant temperature in Si implanted samples. ${ }^{18}$ It is not surprising to observe loops for a heavy implant species such as Sn. Yet, it is surprising to find the complete suppression of loop formation by increasing the implant temperature. Local amorphous regions resulting from dense cascades have been widely predicted by various articles both theoretical ${ }^{23}$ and experimental, ${ }^{24}$ and such regions have been confirmed by TEM. ${ }^{25}$ Amorphous layers generally recrystallize leaving the 
well known end of range loops behind. ${ }^{7}$ Heavy implant species such as Sn are likely to create local amorphous regions which presumably transform into loops during thermal processing. It is well known that the implant temperature determines the amount of dynamic annealing of the implant damage and as a result, elevated temperatures may suppress the formation of local amorphous zones and therefore of loops. Furthermore, dislocation loops are generally created in silicon subjected to amorphizing implants ${ }^{26}$ whereas rod-like defects are usually formed in less-damaged silicon subjected to implants below amorphization doses-the trend from loops to rod-like defects with increasing implant temperatures in our Sn-implanted materials is consistent with decreasing implant damage.

\section{CONCLUSION}

In conclusion, the nature of secondary defects are influenced by implant temperature for heavy ion implantation in $\mathrm{Si}$ in the subamorphous dose regime. We have shown that higher implant temperature can suppress the formation of interstitial loops induced by $\mathrm{Sn}$ implantation in $\mathrm{Si}$, resulting only in rod-like defects in samples implanted at $300{ }^{\circ} \mathrm{C}$. Furthermore, from implants at 8 and $-191{ }^{\circ} \mathrm{C}$, we observed a tendency for smaller loops to form at lower temperature, also for a smaller proportion of these to be faulted. This is attributed to the dense cascades created by the heavy Sn ions resulting in amorphous zones, the formation of which is strongly suppressed due to enhanced dynamic annealing at higher implant temperature.

\section{ACKNOWLEDGMENTS}

One of the authors (J.W-L.) would like to acknowledge the Australian Research Council for financial support under the ARC fellowship program. The authors are indebted to Dr. Bernadette de Mauduit from CEMES-CNRS, Toulouse, for fruitful and interesting discussions related to the characterization of the loops.
${ }^{1}$ R. J. Schreutelkamp, J. S. Custer, J. R. Liefting, W. X. Lu, and F. W. Saris, Mater. Sci. Rep. 6, 275 (1991).

${ }^{2}$ P. Kringhøj, J. S. Williams, and C. Jagadish, Appl. Phys. Lett. 65, 2208 (1994)

${ }^{3}$ S. Fatima, J. Wong-Leung, J. D. Fitz Gerald, and C. Jagadish, Appl. Phys. Lett. 72, 3044 (1998).

${ }^{4}$ S. Fatima, J. Wong-Leung, J. D. Fitz Gerald, and C. Jagadish, Appl. Phys. Lett. 74, 1141 (1999).

${ }^{5}$ S. Coffa, S. Libertino, and C. Spinella, Appl. Phys. Lett. 76, 321 (2000).

${ }^{6}$ D. C. Schmidt, B. G. Svensson, M. Seibt, C. Jagadish, and G. Davies, J. Appl. Phys. 88, 2309 (2000).

${ }^{7}$ K. S. Jones, S. Prussin, and E. R. Weber, Appl. Phys. A: Solids Surf. 45, 1 (1988).

${ }^{8}$ I. G. Salisbury and M. H. Loretto, Philos. Mag. A 39, 317 (1979).

${ }^{9}$ S. M. Davidson and G. R. Booker, Radiat. Eff. 6, 33 (1970).

${ }^{10}$ D. J. Eaglesham, P. A. Stolk, H. J. Gossmann, and J. M. Poate, Appl. Phys. Lett. 65, 2305 (1994)

${ }^{11}$ C. T. Chou, D. J. H. Cockayne, J. Zou, P. Kringhøj, and C. Jagadish, Phys. Rev. B 52, 17223 (1994).

${ }^{12}$ D. J. Eaglesham, P. A. Stolk, H. J. Gossmann, T. E. Haynes, and J. M. Poate, Nucl. Instrum. Methods Phys. Res. B 106, 191 (1995).

${ }^{13}$ J. M. Glasko, R. G. Elliman, J. Zou, D. J. H. Cockayne, and J. D. Fitz Gerald, Appl. Phys. Lett. 73, 838 (1998).

${ }^{14}$ G. Bai and M.-A. Nicolet, J. Appl. Phys. 70, 3551 (1991).

${ }^{15}$ S. B. Herner, H.-J. Gossmann, L. P. Pelaz, G. H. Gilmer, M. Jaraiz, D. C. Jacobson, and D. J. Eaglesham, J. Appl. Phys. 83, 6108 (1998).

${ }^{16}$ L. Pelaz, G. H. Gilmer, M. Jaraiz, S. B. Herner, H.-J. Gossmann, D. J. Eaglesham, G. Hobler, C. S. Rafferty, and J. Barbolla, Appl. Phys. Lett. 73, 1421 (1998).

${ }^{17}$ M. L. Jenkins, D. J. H. Cockayne, and M. J. Whelan, J. Microsc. 98, 155 (1973).

${ }^{18}$ J. Wong-Leung, S. Fatima, C. Jagadish, and J. Fitz Gerald, Defect Diffus. Forum 183-185, 163 (2000).

${ }^{19}$ G. Z. Pan, K. N. Tu, and S. Prussin, Appl. Phys. Lett. 71, 659 (1997).

${ }^{20}$ B. de Mauduit, L. Laânab, C. Bergaud, M. M. Faye, A. Martinez, and A. Claverie, Nucl. Instrum. Methods Phys. Res. B 84, 190 (1994).

${ }^{21}$ A. Claverie, C. Bonafos, A. Martinez, and D. Alquier, Solid State Phenom. 47-48, 195 (1996).

${ }^{22}$ G. Z. Pan, K. N. Tu, and A. Prussin, J. Appl. Phys. 81, 78 (1997).

${ }^{23}$ L. M. Howe and M. H. Rainville, Nucl. Instrum. Methods 182/183, 143 (1981).

${ }^{24}$ M. J. Caturla, T. Diaz de la Rubia, and G. H. Gilmer, Nucl. Instrum. Methods Phys. Res. B 106, 1 (1995).

${ }^{25}$ M. Seibt, J. Imschweiler, and H. A. Hefner, Mater. Res. Soc. Symp. Proc. 262, 1103 (1992).

${ }^{26}$ K. S. Jones, K. Moller, J. Chen, M. Puga-Lambers, B. Freer, J. Berstein, and L. Rubin, J. Appl. Phys. 81, 6051 (1997). 